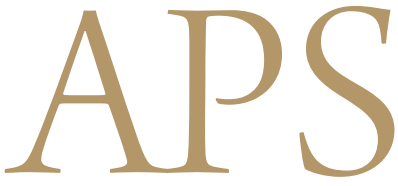

Archives of Plastic Surgery

\title{
De-epithelialized dermal flap for nipple reconstruction: A modified star flap
}

\author{
Su Hyun Lee ${ }^{1}$, Deok Woo Kim ${ }^{1}$, Hi Jin You ${ }^{1}$, Jae A Jung ${ }^{1}$, Na Hyun Hwang ${ }^{1}$, Jae Pil You ${ }^{1}$, \\ Eul Sik Yoon ${ }^{2}$ \\ ${ }^{1}$ Department of Plastic and Reconstructive Surgery, Korea University Ansan Hospital, Ansan; ${ }^{2}$ Department of Plastic and Reconstructive \\ Surgery, Korea University Anam Hospital, Seoul, Korea
}

Background Multiple approaches for nipple reconstruction exist, and none is considered superior to all others. The star flap is one of the most popular methods for nipple reconstruction, but gradual height loss is a major concern. We present a new modification of the star flap that incorporates a de-epithelialized dermal flap, along with the associated surgical results.

Methods We reviewed the medical records of patients who underwent nipple reconstruction using the modified star flap method. The design was different from the conventional star flap in that the lateral wings were changed into a trapezoidal shape and de-epithelialized dermal flaps were added. The patients were followed up at 2, 4, 6, and 12 months postoperatively, and nipple height was measured. The postoperative nipple height achieved using the modified method was compared with that obtained using the traditional method.

Results From February 2013 to June 2017, 32 patients received surgery using the modified star flap, and 18 patients who underwent nipple reconstruction before 2013 comprised the conventional method group. All patients had undergone breast reconstruction with an abdominal tissue-based flap. The mean follow-up period was 14.4 months in the modified method group and 17.3 months in the conventional method group. The mean maintenance of projection at 12 months postoperatively was $56.28 \% \pm 18.58 \%$ in the modified method group, and $44.23 \% \pm 14.15 \%$ in the conventional method group. This difference was statistically significant $(\mathrm{P}<0.05)$.

Conclusions The modified method using a de-epithelialized dermal flap provides reliable maintenance of projection in patients who have undergone abdominal tissue-based breast reconstruction.

Keywords Nipples / Surgical flaps / Mammoplasty
Correspondence: Deok Woo Kim Department of Plastic and Reconstructive Surgery, Korea University Ansan Hospital, 123 Jeokgeum-ro, Danwon-gu, Ansan 15355, Korea

Tel: $+82-31-412-4288$

Fax: +82-31-475-5074

E-mail: deokwookim@gmail.com

This article was presented at the 14 th Congress of Korea-Japan Congress of Plastic and Reconstructive Surgery on June 3-5, 2018, in Pyeongchang, Korea.

Received: 25 Aug 2018 - Revised: 12 May $2019 \bullet$ Accepted: 29 May 2019

pISSN: 2234-6163 • elSSN: 2234-6171 • https://doi.org/10.5999/aps.2018.01011 • Arch Plast Surg 2019;46:324-329

\section{INTRODUCTION}

The nipple-areolar complex (NAC) is an essential structure, the reconstruction of which is a significant component of recreating breast tissue. Patients who undergo both breast and nipple re- construction are more satisfied and have more natural-looking breasts than patients who undergo breast reconstruction alone $[1]$.

Methods for NAC reconstruction include composite grafts and local flaps. Local flap techniques include the star flap, intro- 
duced by Anton et al. [2], which is the most popular method to date. Using three triangular wing flaps, the operator can obtain a moderately projected nipple, and the donor site can be closed primarily. However, the height and volume of the newly reconstructed nipple collapses over time. To overcome these shortcomings, many modified methods, including changes of its design and the use of an acellular dermal matrix graft, have been introduced [3].

Therefore, we developed a new star flap modification that uses de-epithelialized dermal wings for nipple reconstruction and surgical refinement. We analyzed the aesthetic outcomes and any associated complications of patients who underwent reconstruction using this method.

\section{METHODS}

From February 2013 to June 2017, 32 patients who underwent NAC reconstruction using the modified method were analyzed. Our previous method of nipple reconstruction was the star flap or the C-V flap. Therefore, we were able to compare the results of the modified method with those of the conventional method. Nipple height was recorded immediately after the operation and at 2, 4, 6, and 12 months postoperatively, and clinical photographs were taken (Table 1 ). This study was approved by the Institutional Review Board (IRB) of Korea University Ansan Hospital (IRB No. 2019AS0048). Written informed consent was obtained from all patients.

The total number of patients was 32 in the modified star flap group, and 18 in the conventional method group. Twenty-nine patients in the modified method group had undergone breast reconstruction with a free transverse rectus abdominis myocutaneous (TRAM) flap, and three with a deep inferior epigastric

\section{Table 1. Demographic and clinical information of the patients}

\begin{tabular}{lcc|}
\hline Characteristic & $\begin{array}{c}\text { Modified star } \\
\text { flap method } \\
(\mathbf{n}=\mathbf{3 2})\end{array}$ & $\begin{array}{c}\text { Conventional } \\
\text { method } \\
(\mathbf{n}=\mathbf{1 8})\end{array}$ \\
\hline Mean age (yr) & 50.1 & 52 \\
Mean follow-up period (mon) & 14.4 & 17.3 \\
Mean period from breast reconstruction to & 7.4 & 8.5 \\
$\quad$ nipple reconstruction (mon) & & \\
Reconstruction method & 29 & 16 \\
$\quad$ Free TRAM & 3 & 2 \\
$\quad$ DIEP & & \\
Timing of breast reconstruction & 26 & 17 \\
$\quad$ Immediate & 6 & 1 \\
$\quad$ Delayed & 21 & 10 \\
Adjunctive chemotherapy & 3 & 2 \\
Adjunctive radiotherapy & & \\
\hline
\end{tabular}

TRAM, transverse rectus abdominis myocutaneous; DIEP, deep inferior epigastric perforator. artery perforator (DIEP) flap. In the conventional method group, 16 patients had undergone breast reconstruction with a free TRAM flap, and two patients with a DIEP flap. The ratio of the initial nipple projection and projection at follow-up was calculated and compared. Patient demographics, complications, type of breast reconstruction, chemotherapy status, and radiotherapy status were also reviewed.

\section{Surgical technique}

The NAC reconstruction was performed after completion of adjunctive therapy, and if no adjunctive treatment was scheduled, NAC reconstruction was performed at 2 months after breast reconstruction.

The modified star flap that we designed was different from the conventional star flap in that the lateral wings of the flap were changed from a triangular shape to a trapezoid, and de-epithelialization was done on both ends of the lateral wings. In essence, the design was composed of equal segments, which were 10 to $12 \mathrm{~mm}$, depending on the size of the contralateral nipple (Fig. 1). There were two different de-epithelialized areas: triangular and fan-shaped. Triangular dermal flaps located on both ends of the lateral wings were rolled into the column of the neo-nipple for volume maintenance. The fan-shaped de-epithelialized areas were not elevated with the flaps. When elevating the flap, unlike conventional methods, there was no need to attach much fat tissue under the flap, because dermal flaps filled the volume of the nipple instead of fat tissue.

After de-epithelialization and flap elevation, the triangular dermal flaps were rolled inside the column of the nipple. This procedure filled the inner cavity of the nipple with resilient dermis, instead of collapsible fat, as in conventional methods. When closing the donor site, the fan-shaped dermal flaps were gathered from both sides to form a semicircle at the bottom of the

\section{Fig. 1. Modified star flap design}

The skin was incised along the thick line, and the shaded area was de-epithelialized. I indicates the length of each segment, which was usually 10-12 mm, depending on the height of the contralateral nipple.

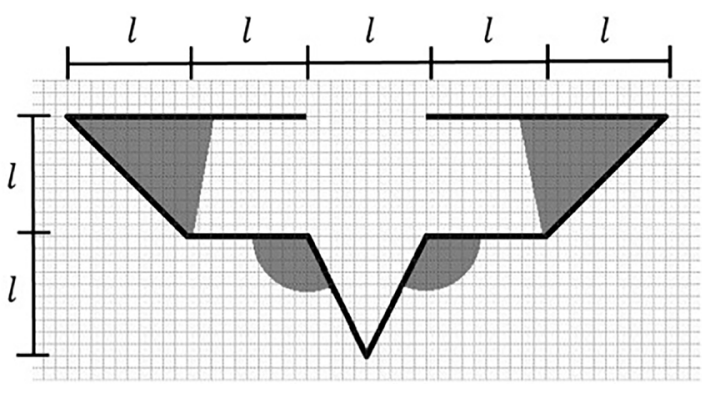




\section{Fig. 2. Intraoperative photographs}

(A) Placing the design. (B) De-epithelialization. (C) Rolling the lateral dermal flap into the neo-nipple column. (D) Completion of the neo-nipple.
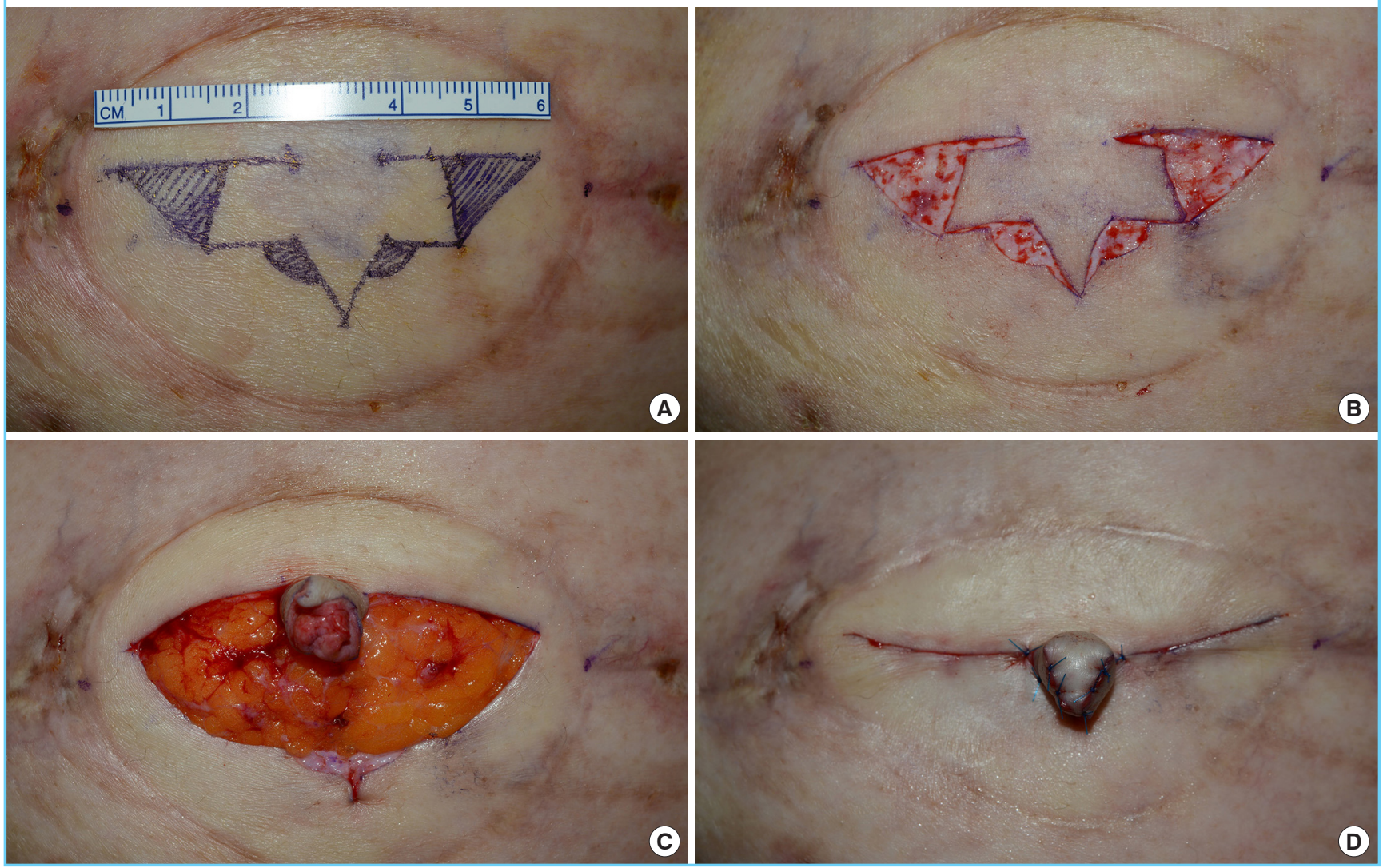

nipple. The two fan-shaped dermal areas that formed the base of the nipple prevented the nipple from collapsing (Fig. 2). The lower skin flaps were subcutaneously undermined, advanced, and closed. Antibiotic ointment was applied on the reconstructed nipple and punched-out foam dressing was applied for protection. A nipple protector was maintained postoperatively for 8 weeks.

At 2 months after the procedure, the NAC was tattooed with a natural nipple color. Some patients required several sessions, depending on the color preservation.

\section{Assessment}

The reconstructed nipple height was measured in millimeters from the base to the furthest protruding tip of the nipple at the time of surgery and at 2, 4, 6, and 12 months postoperatively. The maintenance of nipple projection was calculated as the percentage of final height relative to the initial height of the nipple. The following equation was used:

$$
\text { Maintenance }=\frac{(\text { Follow-up projection })}{(\text { Initial projection })} \times 100(\%) \text {. }
$$

Statistical analysis was performed using SPSS version 12.0 (SPSS Inc., Chicago, IL, USA).

\begin{tabular}{|c|c|c|}
\hline Outcome & $\begin{array}{l}\text { Modified star } \\
\text { flap method }\end{array}$ & $\begin{array}{l}\text { Conventional } \\
\text { method }\end{array}$ \\
\hline Initial projection $(\mathrm{mm})$ & $10.6 \pm 4.26$ & $11.3 \pm 1.79$ \\
\hline Follow-up projection (mm) & $5.83 \pm 2.96^{\mathrm{a})}$ & $5.11 \pm 2.52$ \\
\hline Maintenance (\%) & $56.28 \pm 18.58^{\text {a) }}$ & $44.23 \pm 14.15$ \\
\hline Complications & $1(3.1)$ & $1(5.6)$ \\
\hline
\end{tabular}

\section{RESULTS}

The mean initial projection of the nipple was $10.6 \pm 4.26 \mathrm{~mm}$ in the modified method group, and $11.3 \pm 1.79 \mathrm{~mm}$ in the conventional method group. The projection in the modified method group fell to $5.83 \pm 2.96 \mathrm{~mm}$ after an average of 14.4 months, and the projection in the conventional method group fell to $5.11 \pm$ $2.52 \mathrm{~mm}$ after an average of 17.3 months. The mean ( \pm standard deviation) maintenance of projection was $56.28 \% \pm 18.58 \%$ in the modified method group, and $44.23 \% \pm 14.15 \%$ in the conventional method group (Table 2). The Student t-test showed that there was a statistically significant difference between the 


\section{Fig. 3. Postoperative photographs}

(A) Immediate postoperative photograph. (B) One year and 8 months postoperatively. (C) Initial photograph. (D) Seven months postoperatively. (E) Initial photograph. (F) Nine months postoperatively. (G) Three months postoperatively. (H) One year postoperatively.
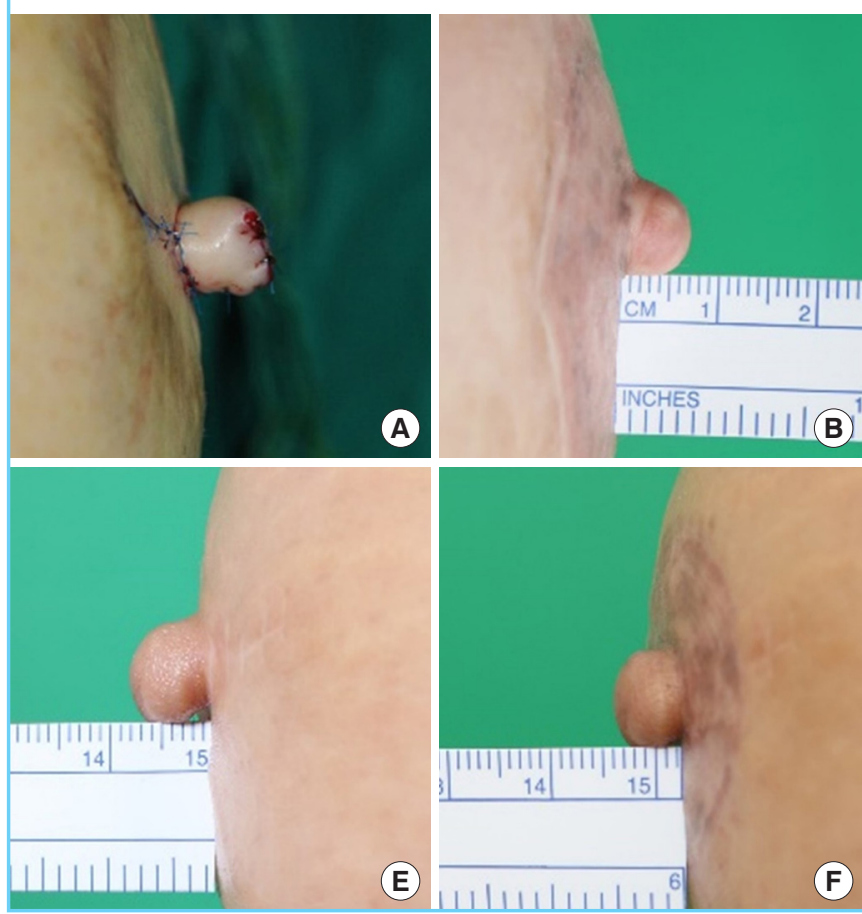

(F)

two groups $(\mathrm{P}<0.05)$. Postoperative photographs of the modified group are shown in Fig. 3.

\section{DISCUSSION}

Following breast reconstruction, most patients opt for nipple reconstruction because it can restore the symbol of breast tissue [4]. However, collapse of the reconstructed nipple is frustrating for both patients and surgeons [5]. Numerous methods for NAC reconstruction exist, although no single method is currently considered to be superior; therefore, new methods are being developed [6]. The ultimate goal is to optimize the surgical approach and provide better maintenance than is possible with existing methods.

Our technique is not the first to apply a de-epithelialized dermal flap [7]. Temiz et al. [8] introduced a modification of the C-V flap technique, which utilizes a triangular dermal flap on each wing to fill the neo-nipple. Saleh and Mohammed [7] presented another method that completely de-epithelializes one of the lateral wings and rolls it into a neo-nipple. Those variant methods - as well as ours - use dermal flaps because of the belief that dermal tissues are durable and less likely to shrink. In addition, an experimental study proved that the dermis is the layer of the skin with the greatest mechanical stiffness [9].

We examined the reconstructed nipples. A patient who under-

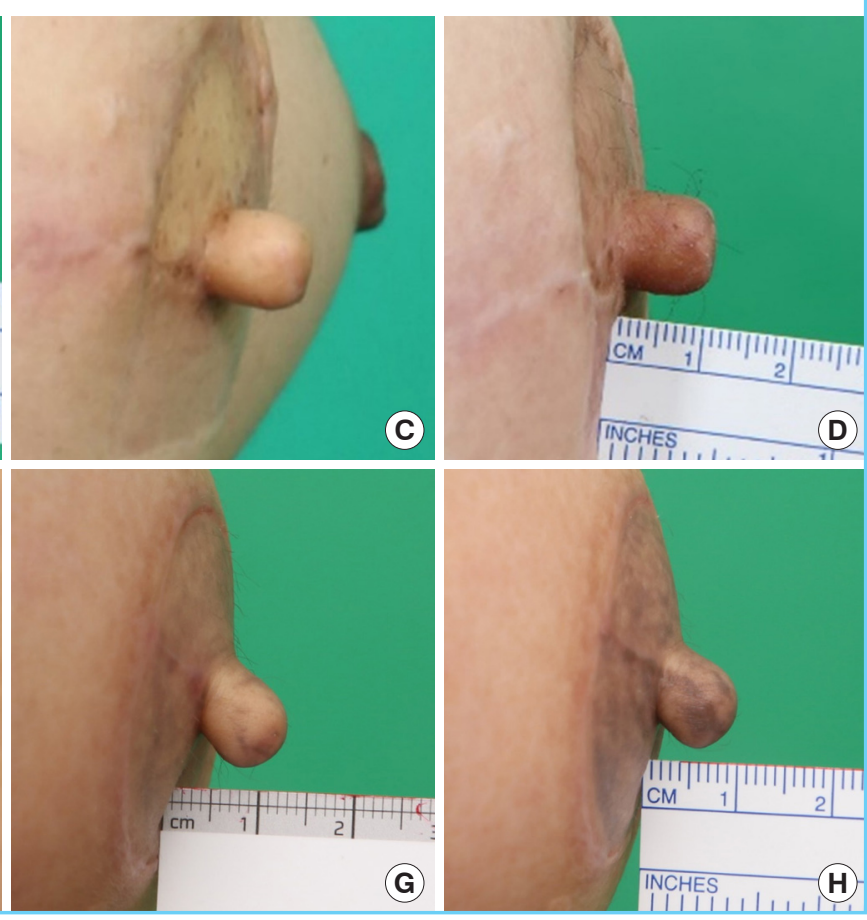

\section{Fig. 4. Composition of the neo-nipple}

The W-shaped white linear tissue in the cross-sectioned neo-nipple, thought to be dermal tissue, was well maintained (white arrows: direction in which the dermal flap was rolled).

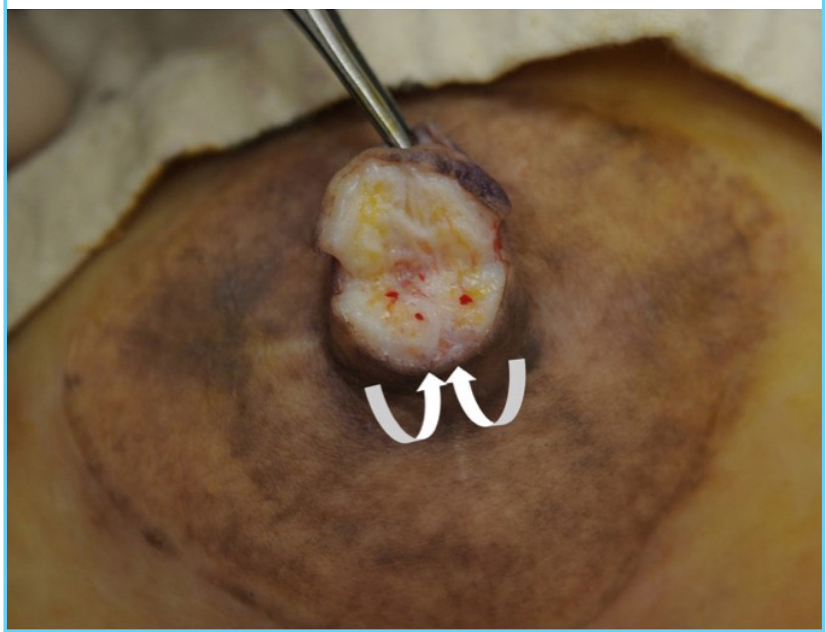

went nipple reconstruction 11 months prior visited our clinic due to high projection of the nipple and wanted nipple reduction. The height of the reconstructed nipple was $9 \mathrm{~mm}$, while that of the nipple on the contralateral breast was $6 \mathrm{~mm}$. Under local anesthesia, a wedge resection was carried out on the column of the nipple to a height of $6 \mathrm{~mm}$. During the procedure, we were able to observe $\mathrm{W}$-shaped white dermal tissue (the 


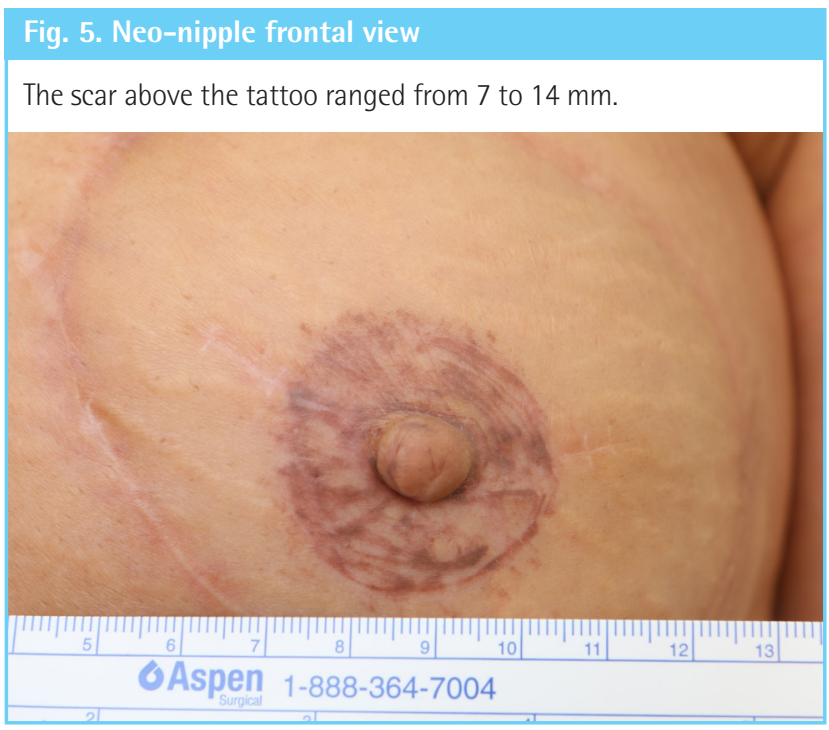

rolled-in triangular dermal flaps) in the cross-section of the nipple. This observation confirmed that the dermal flaps were well maintained inside the neo-nipple (Fig. 4).

The properties of skin should be considered in nipple reconstruction. Skin that originates from the abdomen (TRAM or DIEP) has a thinner dermis layer than skin from the back (in the latissimus dorsi flap) [10]. In regard to TRAM flaps, it is often necessary to fill the volume inside the nipple with resilient materials. Therefore, there are several methods that incorporate dermal flaps or acellular dermal matrix instead of dermis $[11,12]$. Acellular dermal matrix is a good alternative to autologous dermis [13]. However, the associated costs are high and it can cause foreign body-associated reactions $[14,15]$. In this context, the dermal flap used in our technique is adequate for patients who undergo abdominal skin-based breast reconstruction.

Although not shown in this article, in cases where a latissimus dorsi flap was used for breast reconstruction cases, the de-epithelialized triangular dermal flap was used infrequently due to its thick dermis. In such cases, the size of dermal flaps beside the lateral wings could be reduced to minimize horizontal scarring.

We hypothesize that the narrower base of the nipple, rather than the tip, is important both for creating an omega-shaped nipple and for maintaining projection. We observed that the height was reduced as the base of the nipple gradually widened. Considering the fact that the base gradually widened, we intentionally made the base narrower than the tip.

However, we noted a few shortcomings of our technique. In the modified star flap method, the lateral wings are longer than in other methods, since dermal flaps are used at both ends. After closing the donor site, the horizontal scars are longer than those of conventional methods and are visible over the tattoo (Fig. 5). The length not veiled by tattooing is around $1 \mathrm{~cm}$, but patients rarely complained about the scar. Since patients with short areolar diameters suffer from long visible scars, this should be taken into consideration when determining the size of dermal flaps to be raised for the lateral wing.

There were some limitations in our study. First, the sample size of the conventional method group was small. In the future, data collected and analyzed at multiple centers could provide additional insights applicable for the general population. Second, only the results of abdomen-based flap cases were analyzed. We were therefore unable to demonstrate whether this approach can be applied in cases of previous implant-based breast reconstruction.

The modified star flap is a simple and reliable method that can improve the long-term maintenance of projection. This approach is cost-effective and shows aesthetically satisfactory results.

\section{NOTES}

\section{Conflict of interest}

No potential conflict of interest relevant to this article was reported.

\section{Ethical approval}

The study was approved by the Institutional Review Board of Korea University Ansan Hospital (IRB No. 2019AS0048) and performed in accordance with the principles of the Declaration of Helsinki. Written informed consents were obtained.

\section{Patient consent}

The patients provided written informed consent for the publication and the use of their images.

\section{Author contribution}

Study concept and design: Kim DW. Data analysis and interpretation, drafting of the manuscript: Lee SH. Critical revision of the manuscript for important intellectual content: Yoon ES, You HJ, Hwang NH, You JP, Jung JA.

\section{ORCID}

Su Hyun Lee https://orcid.org/0000-0001-5846-392X

Deok Woo Kim https://orcid.org/0000-0003-3382-560X

Hi Jin You https://orcid.org/0000-0002-9997-2736

Jae A Jung https://orcid.org/0000-0002-4804-9634

Na Hyun Hwang https://orcid.org/0000-0001-8784-7383

Jae Pil You https://orcid.org/0000-0002-0701-7046

Eul Sik Yoon https://orcid.org/0000-0001-5734-6625 


\section{REFERENCES}

1. Momoh AO, Colakoglu S, de Blacam C, et al. The impact of nipple reconstruction on patient satisfaction in breast reconstruction. Ann Plast Surg 2012;69:389-93.

2. Anton MA, Eskenazi LB, Hartrampf CR Jr. Nipple reconstruction with local flaps: star and wrap flaps. Perspect Plast Surg 1991;5:68-78.

3. Bramhall RJ, Thiruchelvam PT, Concepcion M, et al. Use of acellular dermal matrix (ADM) in nipple reconstruction: the 'central-pillar technique'. Gland Surg 2017;6:394-8.

4. Weissler EH, Schnur JB, Lamelas AM, et al. The necessity of the nipple: redefining completeness in breast reconstruction. Ann Plast Surg 2017;78:646-50.

5. Jabor MA, Shayani P, Collins DR Jr, et al. Nipple-areola reconstruction: satisfaction and clinical determinants. Plast $\mathrm{Re}$ constr Surg 2002;110:457-63.

6. Nimboriboonporn A, Chuthapisith S. Nipple-areola complex reconstruction. Gland Surg 2014;3:35-42.

7. Saleh DB, Mohammed PD. The double-breasted dermal flap in nipple reconstruction. J Reconstr Microsurg 2013;29: 421-2.

8. Temiz G, Yesiloglu N, Sirinoglu H, et al. A new modification of C-V flap technique in nipple reconstruction: rolled triangular dermal-fat flaps. Aesthetic Plast Surg 2015;39:173-5.
9. Crichton ML, Donose BC, Chen X, et al. The viscoelastic, hyperelastic and scale dependent behaviour of freshly excised individual skin layers. Biomaterials 2011;32:4670-81.

10. Whitton JT, Everall JD. The thickness of the epidermis. Br J Dermatol 1973;89:467-76.

11. Park GY, Yoon ES, Cho HE, et al. Acellular dermal matrix as a core strut for projection in nipple reconstruction: approaches for three different methods of breast reconstruction. Arch Plast Surg 2016;43:424-9.

12. Rao SS, Seaman BJ, Davison SP. The acellular dermal matrix onlay graft for areolar reconstruction. Ann Plast Surg 2014; 72:508-12.

13. Lee JH, Kim HG, Lee WJ. Characterization and tissue incorporation of cross-linked human acellular dermal matrix. Biomaterials 2015;44:195-205.

14. Winocour S, Saksena A, Oh C, et al. A systematic review of comparison of autologous, allogeneic, and synthetic augmentation grafts in nipple reconstruction. Plast Reconstr Surg 2016;137:14e-23e.

15. Krishnan NM, Chatterjee A, Van Vliet MM, et al. A comparison of acellular dermal matrix to autologous dermal flaps in single-stage, implant-based immediate breast reconstruction: a cost-effectiveness analysis. Plast Reconstr Surg 2013;131: 953-61. 